les machines de laiterie n'agitent pas le lait trop violemment. Il peut en résulter une homogénéisation partielle qui peut entraîner une rancidité plus ou moins prononcée.

Au point de vue théorique, ce travail ouvre un certain nombre de perspectives pour une meilleure connaissance du lait et des facteurs à la base de sa formation. Il ne semble pas qu'il existe une relation entre la teneur du lait en lipase et sa faculté de coaguler par la présure.

\title{
ACTION DU CUIVRE, DE L'ACIDE LACTIQUE ET DE LA TEMPÉRATURE SUR L'AUTO-OXYDATION DE LA MATIËRE GRASSE DU BEURRE ET DU SAINDOUX (1)
}

\author{
par K. E. WRIGHT (2) et O. R. OVERMAN (3) \\ INTRODUCTION.
}

Il y a de nombreuses années qu'on sait en industrie laitière que des saveurs suiffeuses et rances dans les produits laitiers peuvent être attribuées à certaines conditions dans lesquelles les produits sont fabriqués. Le stade suiffeux de la détérioration a été observé dans le lait condensé et était, dans ce cas, souvent associé à la fabrication dans des vacuums en cuivre. On a souvent observé que l'acidité de la crème a une forte influence sur la qualité du beurre qui en provient. On a aussi compris que la chaleur est un de ces facteurs, qu'elle favorise spécialement l'hydrolyse due aux bactéries, quoique son effet direct ne soit pas entièrement connu. On admet que la saveur suiffeuse est une détérioration d'oxydation accompagnant le développement de la rancidité. Alors que l'effet de ces trois facteurs : chaleur, acidité et cuivre, sûr par oxydation, est partiellement connu, la connaissance de leurs effets relatifs est loin d'être complète.

Lorsque les matériaux différents servant à la construction des appareils, tels que le verre, les alliages et le cuivre, auront été étudiés pour leurs qualités relativement à ce point de vue, cette étude sera utile parce qu'elle donnera une représentation graphique des effets séparés ci-dessus mentionnés. Il sera également utile d'essayer d'établir la méthode la plus convenable de fabrication du beurre, étant donné les différentes méthodes en usage. C'est la nécessité de cette connaissance qui nous a conduits à déterminer l'influence relative de quelques-uns des facteurs de la détérioration des produits laitiers.

Quand une graisse, telle que la matière grasse du beurre ou le saindoux, contenant des acides gras non saturés, comme l'acide

(1) Cette étude est extraite de la thèse sjumize par K. E. Wrightà la " Graduate School " de l'Université d'Illinois pour le titre de "Master of Science n en Economie Laitière, 1927.

(2) Professeur adjoint, Recherches laitières, Station d'Essajs agricoles, Amherst (Mass.).

(3) Professeur adjoint de Chimie Laitière, Université de J'Mlinois, Urbana (III). 
oléique, commence à devenir suiffeuse, une modification chimique définie, marquée, a commencé : c'est l'oxydation. En fait, cette modification est si prononcée qu'en l'espace d'un moment la graisse peut de neutre devenir suiffeuse et commencer à absorber l'oxygène à une vitesse qui est très facilement mesurée. C'est spécialement le cas quand la graisse est en présence de l'oxygène à la température de l'eau bouillante.

La durée de la période précédant l'absorption active de l'oxygène par la graisse du beurre dépend des conditions dans lesquelles le produit laitier a été fabriqué et conservé. Cette période est connue sous le nom de période d'induction ou période d'auto-oxydation. Les conditions les plus importantes peuvent en être indiquées brièvement. L'activité bactérienne précédant la fabrication des produits laitiers a pour effet l'hydrolyse ou attaque d'une partie de la matière grasse. Ensuite, pendant la fabrication de ces produits, cette petite quantité de graisse attaquée est oxydée en produits qui paraissent agir catalytriquement en oxydant encore plus la masse restante de la graisse pendant la période d'induction. Cette oxydation initiale est accélérée par une augmentation de la température, par la présence d'acide lactique et particulièrement par le contact ayec des surfaces en cuivre. Ainsi, quoique des produits laitiers semblent être relativement semblables en qualité quand on les examine, et ne peuvent pas être différenciés définitivement sur la base des résultats obtenus par les méthodes officielles d'analyse des matières grasses, leur tendance à l'oxydation varie nettement suivant le traitement subi par elles antérieurement.

Faisant suite à la période d'induction, c'est-à-dire à la période d'oxydation naissante, il y a une absorption soudaine et rapide de l'oxygène par la matière grasse, accompagnée par des changements marqués dans la saveur et l'apparence. Ce phénomène permet de déterminer la fin de l'oxydation naissante et le commencement de la détérioration rapide. La période d'induction fut déterminée par le temps écoulé entre l'exposition de la graisse à l'oxygène à la température employée et le début de l'absorption rapide de l'oxygène par la graisse.

En recherchant une méthode pour effectuer cette détermination d'après les principes ci-dessus, on trouva que celle de HoLM et Gremnank (1) était d'une application très facile. Leur appareil permettait d'agiter la graisse en présence d'oxygène à la température de $95^{\circ} \mathrm{C}$. Les mêmes expérimentateurs (2) ont, depuis, décrit une autre méthode pour mesurer la période préliminaire d'oxydation, qui

(1) G. E. Howm et G. R. Greenbank. - Congrès International de Laiterie, 2, 1253, 1923.

(2) G. R. Greenbank et G. E. Holm, - Analytical Edition Ind. and Eng. Chem., 2, 9, 1930. 
consiste à déterminer le temps nécessaire pour réduire le bleu de méthylène dans une graisse non saturée, par l'action d'une forte lumière. Les résultats obtenus par l'ancienne méthode, telle qu'elle a été employée dans la présente étude, restent intéressants, quoiqu'il ne paraisse pas utile d'améliorer la méthode elle-même pour un usage ultérieur.

\section{PARTIE EXPÉRIMENTALE.}

La graisse fut purifiée par simple décantation à une température modérée $\left(50^{\circ}-75^{\circ}\right.$ C.). Un appareil, construit d'après la description donnée par Holm et GreEnBank dans leur première méthode, fut employé pour l'oxydation. Dans ces essais, $325 \mathrm{gr}$. de graisse furent employés. La graisse fut agitée avec une quantité déterminée d'oxygène dans un flacon étanche à l'air à l'aide d'un agitateur scellé au mercure actionné par moteur. La légère absorption initiale d'oxygène fut déterminée par l'emploi d'un manomètre à eau, tandis que l'absorption régulière ultérieure fut mesurée par une burette à gaz de 1 litre. La burette était elle-même maintenue à niveau par un contrepoids, qui permettait les lectures une heure après le commencement de l'absorption. On pouvait ainsi calculer la durée de l'absorption initiale par la lecture, sans observer constamment le manomètre.

On observa que les périodes d'oxydation initiale de la graisse du beurre pure préparée par cette méthode ne différaient pas d'une façon appréciable de celles de la même graisse oxydée en présence d'humidité visible ou en présence d'extrait sec de lait sans matière grasse. C'est pourquoi la méthode de purification par simple décantation n'influença pas d'une façon perceptible la période initiale d'induction de l'oxydation.

A mesure que notre étude progressait, on nota que la graisse du beurre fraîche pouvait être exposée à l'oxygène pur pendant 16 à 30 heures avant qu'une absorption active de l'oxygène n'eût lieu, au lieu de 3 à 6 heures, comme l'avaient indiqué Holm et Grennbank (1). On supposa que cette différence était due à une modification possible dans la méthode de purification. Il semble que la méthode de purification employée par eux était plus rigoureuse. Par exemple, avec l'entraînement à la vapeur d'eau pour enlever les acides gras libres, il y a à craindre l'hydrolyse des glycérides, qui rendrait la graisse plus susceptible d'être oxydée.

Pour des raisons qui nous échappent, cette période trouvée pour de la graisse du beurre fraîche fut influencée par des facteurs qui causèrent des différences dans les périodes d'induction des doubles des mêmes échantillons. Ces variations furent si grandes qu'elles rendirent incompréhensible la différence à laquelle on aurait pu

(1) G. E. Holm et G. R. Greenbank, - Congrès International de Laiterie, 2, 1253, 1923. 
ordinairement s'attendre en raison de l'âge du produit ou des accélérateurs ci-dessus indiqués. Comme ces différences semblaient décroître avec la diminution de la période d'induction, l'étude fut continuée avec le saindoux, qui a des périodes d'induction courtes, en raison de sa plus grande proportion d'acides gras non saturés.

Ainsi, quoique la graisse du beurre frâiche ne fut pas utilisée comme on l'avait prévu, l'utilisation du saindoux donna des résultats qu'on suppose être tout à fait caractéristiques de la façon dont se serait comportée la graisse de beurre. Cette analogie paraît particulièrement justifiée quand les oxydations du saindoux furent comparées avec les résultats obtenus par l'oxydation de vieille graisse du beurre.

Les graphiques suivants sont tracés avec les chiffres obtenus pour montrer la vitesse d'absorption de l'oxygène dans les différentes conditions indiquées.

Dans la planche 1 , les deux courbes de droite donnent les résultats obtenus par l'oxydation de la graisse du beurre fraîche en présence d'oxygène à $96^{\circ} \mathrm{C}$. Les résultats pour les courbes numérotées $21,13,14$ et 15 furent obtenus avec un échantillon composé de déchets de beurre ayant au moins 2 ans d'âge. Les périodes d'induction relevées pour cet échantillon montrent qu'il a été bien plus sujet à l'oxydation qu'on aurait pu le supposer. Les courbes 18 et 19 montrent l'effet de l'addition de 1 pour cent d'acide lactique à une partie de l'échantillon. Les courbes 16,17 et 21 montrent l'effet marqué du cuivre pur comme catalyseur sur une autre partie. Ces derniers résultats montrent que les différences entre des échantillons en double de vieux beurre sont très légères et elles contrastent considérablement avec les variations entre des échantillons en double de bon beurre, comme le montrent les deux courbes de droite aux heures 16 et 32 .

Les effets relatifs du cuivre (courbes 174,175$)$ (180, cuivre avec air) et acide lactique (courbes 179, 176) sur une marque de saindoux très connue sont montrés dans le graphique II. Sont également montrés les résultats de l'oxydation dans l'air (177, 178 et 180 : cuivre et air). Il est significatif au point de vue de la comparaison que le rapport entre les périodes d'induction résultant de l'effet du cuivre et de l'acide lactique soient les mêmes pour le saindoux et le vieux beurre ; on peut done à juste titre considérer les effets du cuivre et de l'acide lactique sur le saindoux comme une base de leur effet relatif sur le beurre.

Les courbes dans la planche III furent obtenues par l'oxydation de saindoux récemment fondu. Comme on pouvait s'y attendre, la période d'induction pour l'essai de contrôle (courbes 181, 182) est quelque peu plus longue que pour l'échantillon plus vieux, quoique bon, étudié dans la planche II. Est également montré, l'effet du cuivre et de la température. 


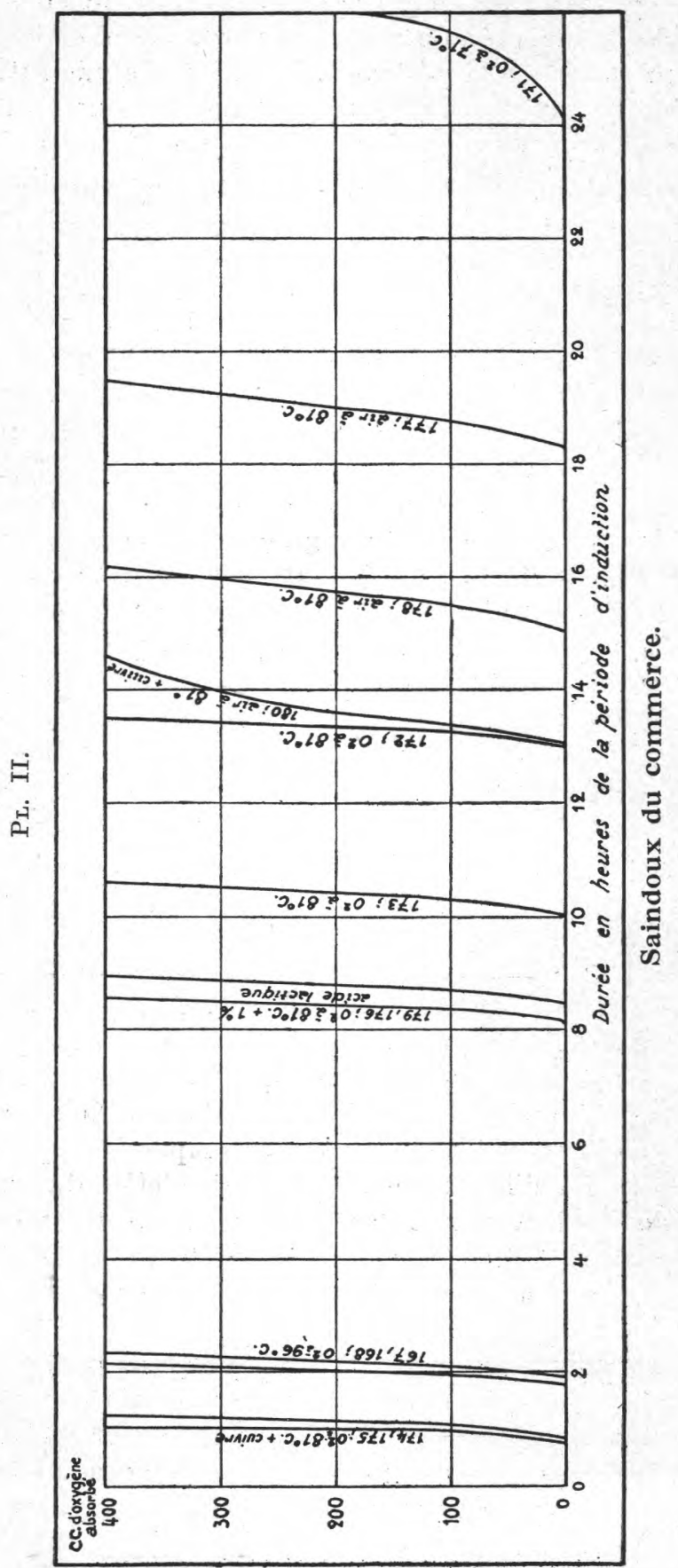





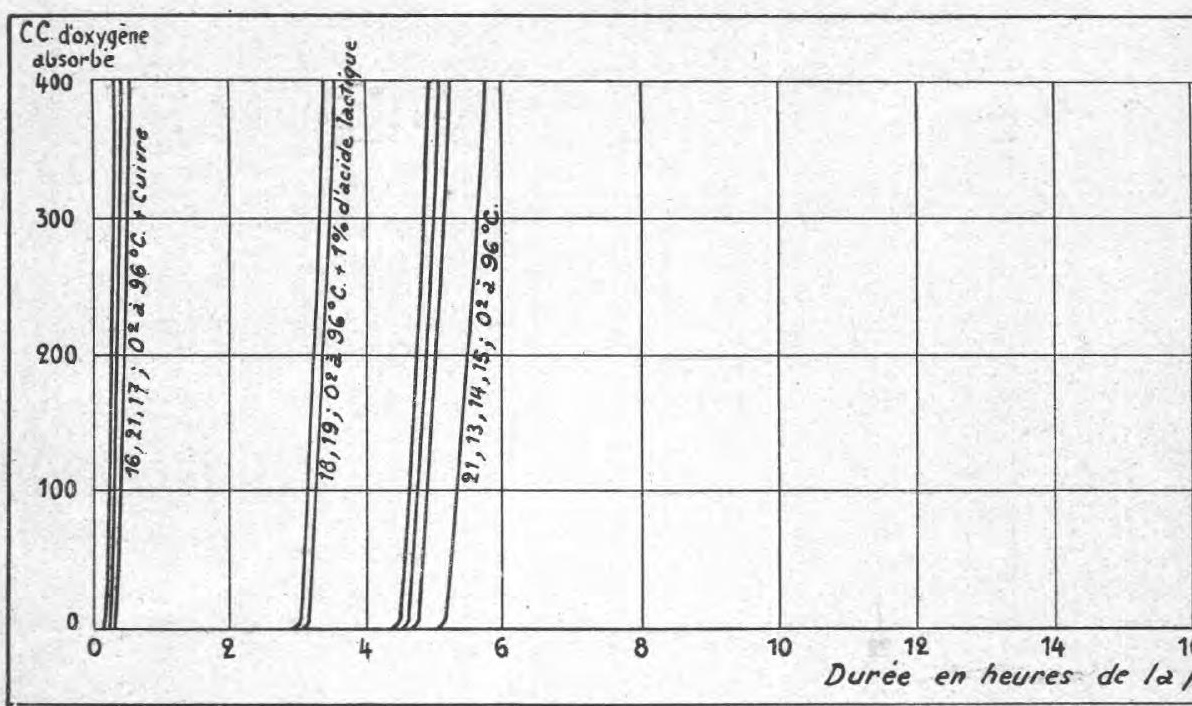

Action du cuivre et de 1'acide lactique sur du vieux beurre

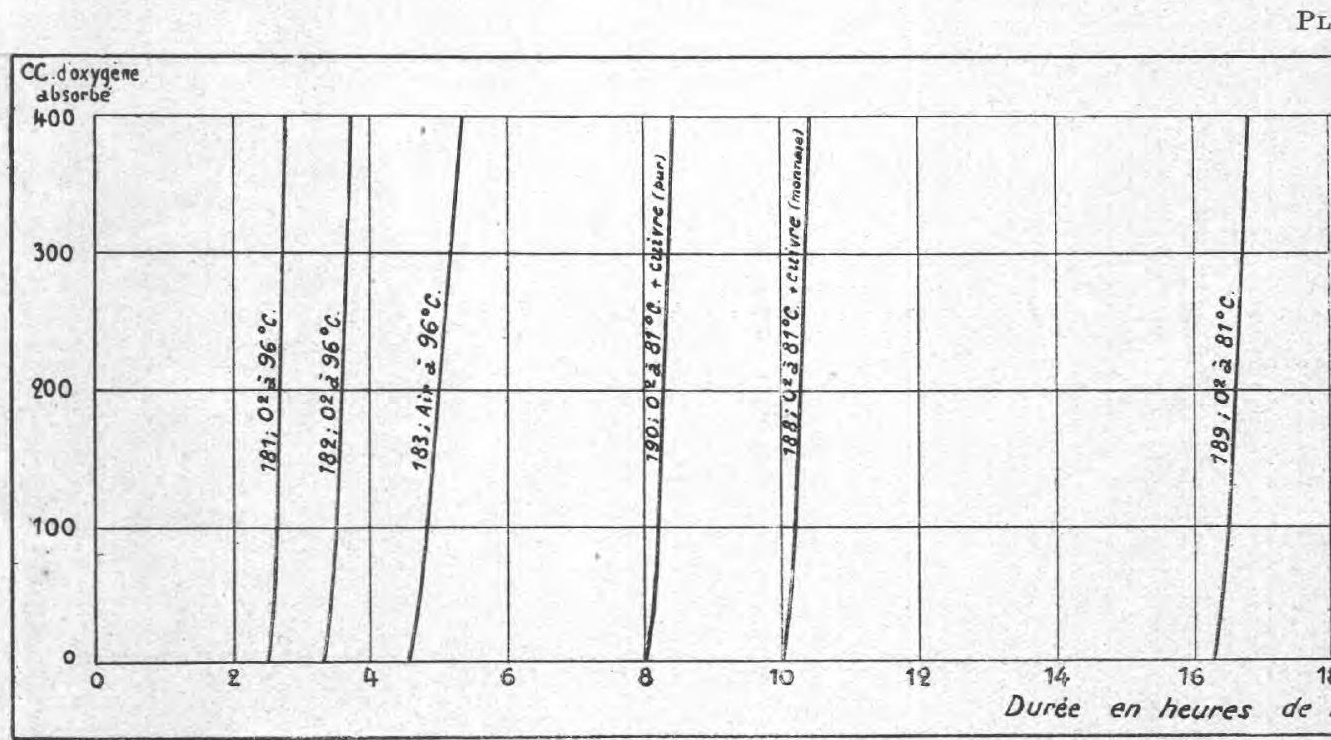

Saindoux fr 


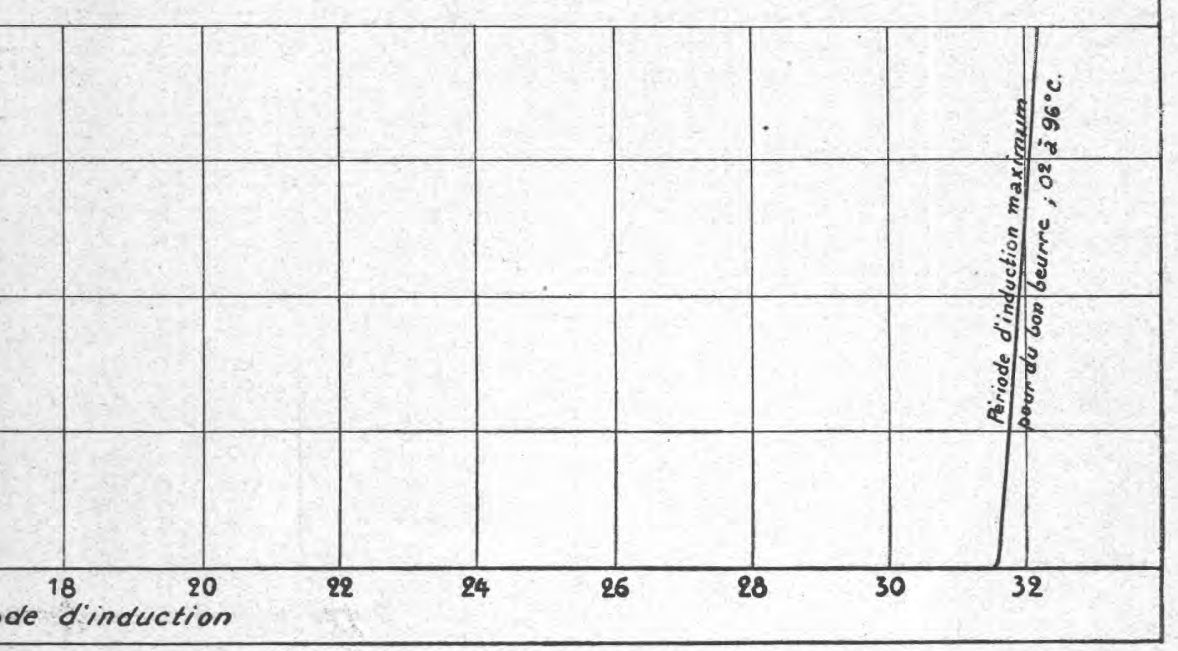

auche). - Périodes d'induction de bons beurres (à droite).

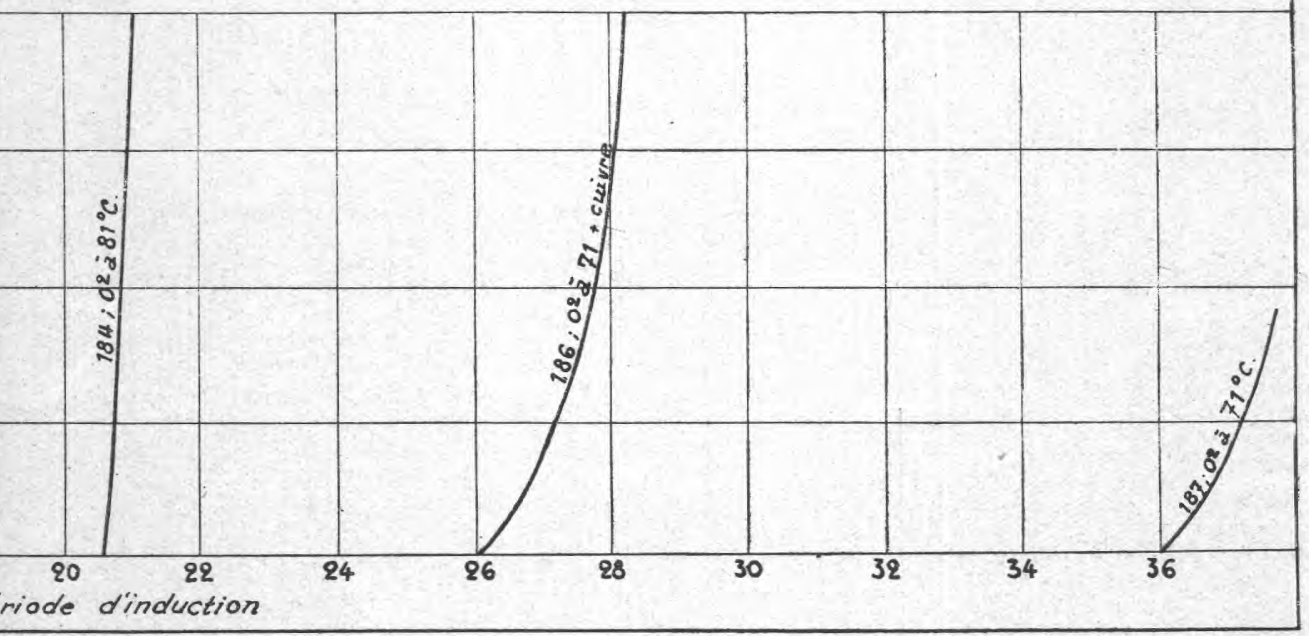

t neutre. 

Le graphique de la pl. IV montre complètement l'effet relatif des diverses températures de $96^{\circ}$ à $61^{\circ} \mathrm{C}$.En supposant des lignes imaginaires pour des températures plus basses, le lecteur pourra se faire une idée approximative de la vitesse de l'oxydation initiale à des températures

PL. IV.

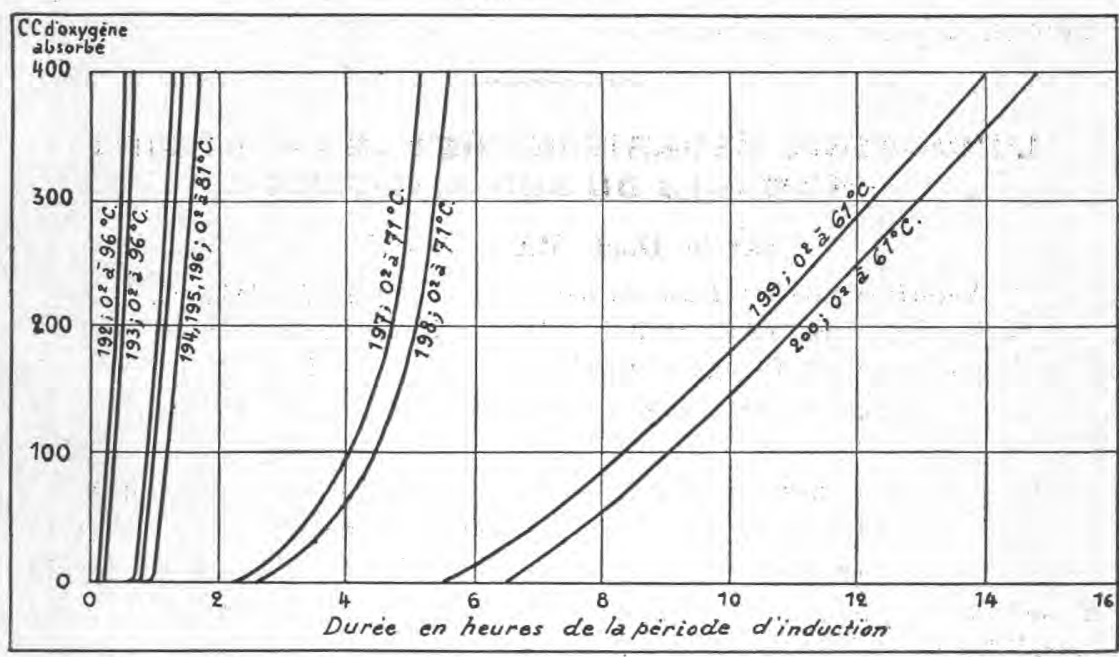

Action de la température sur du vieux saindoux neutre.

du magasinage frigorifique pour cet échantillon. Le saindoux.avec lequel ces chiffres furent obtenus était très ancien, ce qui explique la courte période d'induction (courbes 192 et 193). De nouveau ici, le lecteur est prié de substituer dans son esprit un échantillon de bon beurre demandant plusieurs heures pour la période d'induction et d'essayer de supposer une courbe imaginaire pour l'oxydation probable à basses températures. Quoique l'impossibilité de mesurer des oxydations ä basse température soit évidente, la signification probable peut être imaginée approximativement.

\section{RÉSUMÉ ET CONCLUSIONS.}

1. La faculté de conservation de la graisse et la durée de la période d'induction précédant l'oxydation active de la graisse sont déterminées par le traitement préalable du produit.

2. La période d'absorption rapide de l'oxygène est précédée par tne période d'auto-oxydation qui diminue avec l'âge suivant les agents catalytiques fournis par les bactéries et par le cuivre, l'acidité et la température.

3. Les observations montrent que les oxydations du beurre et du saindoux sont influencées par les facteurs cuivre, acidité et température, d'une manière analogue. 
4. On a trouvé que le cuivre a un effet plus prononcé sur l'oxydation que l'acidité et la température.

5. L'action de la température augmente avec l'élévation de celle-ci.

6. L'acidité a une action nette comme accélérateur de l'oxydation, quoique moins marquée que celle du cuivre ou celle de l'augmentation de la température.

\title{
L'INDUSTRIE BEURRIËRE CHEZ LES PASTEURS NOMADES DU SUD-ALGÉRIEN
}

\author{
par le Doct. BENDANOU \\ Vétérinaire de $1^{\text {re }}$ classe du Service de l'Elevage de l'Algérie, \\ Ancien Répétiteur de Zootechnie â l'Ecole Nationale d'agriculture de \\ Montpellier, Chargé de Missions dans le Territoire du Sud. \\ (Communication faite à l'Office Colonial de l'Algérie)
}

La note que nous présentons sur l'industrie beurrière chez les pasteurs nomades du Sud algérien n'est qu'une contribution à l'étude de l'industrie pastorale en Algérie et au Maroc. Et l'industrie pastorale ne se résume pas dans l'élevage du mouton exclusivement, mais dans l'exploitation la plus rationnelle de tout ce qui dérive de cet élevage. Il est certain que les produits, tels que la laine, la peau, les cornes, les intestins, ne peuvent pas être utilisés totalement sur place ; l'industrie textile, la tannerie sont encore réservées à la Métropole, déjà outillée pour cela ; mais il est d'autres produits, tels que le lait, périssable au plus haut degré, que l'on ne peut exporter, et qui sont déjà l'objet d'une industrie locale.

Les pasteurs nomades tirent du lait ce qu'ils peuvent, mais n'en tirent pas tout ce que ce produit devrait nécessairement donner. Il appartient aux Occidentaux, qui ont assuméla tâche d'enseigner à nos sujets musulmans les meilleures méthodes d'exploitation du troupeau, de les guider et de les éclairer. Leurs procédés, si primitifs qu'ils puissent paraitre, sont le fruit d'unenpratique séculaire et sont rationnels, mais l'indigène stagne ; il ne s'agit que de lui ouvrir la voie et de le pousser à progresser. Là, se pose une question : devons-nous le supplanter, prendre sa place, le déposséder en un mot, ou bien devons-nous, armés de la patience du pédagogue, de l'éducateur, essayer de vaincre sa force d'inertie ? La vérité réside entre ces deux alternatives. L'indigène ne doit pas, à notre contact, se voir ruiné ou réduit à devenir notre salarié ; il doit devenir notre collaborateur et notre associé.

Ce n'est pas l'heure où le monde musulman est agité que nous devons le libérer de toute attache; le jour où l'indigène, jusqu'ici 